ICMB21

\title{
The effect of changes in air humidity on historic tapestries
}

\author{
Pedro Máximo Rocha ${ }^{\mathrm{a}}$, Dina D’Ayala ${ }^{\mathrm{a}}$, Hector Altamirano-Medina ${ }^{\mathrm{a}}$, Constantina Vlachou-Mogire ${ }^{\mathrm{b}}$, \\ Levente Klein ${ }^{\mathrm{c}}$, Alejandro Schrott ${ }^{\mathrm{d}}$ \\ aUniversity College London, London, UK \\ bHistoric Royal Palaces, London, UK \\ c IBM T.J. Watson Research Center, New York, USA \\ d Stony Brook University, New York, USA
}

\begin{abstract}
Historic tapestries are textile artworks usually found hanging inside historic buildings. They form part of the European heritage and have been exposed for centuries to diverse environmental conditions some of which have permanently damaged them. To inform better and appropriate conservation practices, their physical behavior when exposed to environmental changes must be understood.

The research conducted aimed to assess how the internal environment conditions in a historic building affects the tapestries' structures and produce change in their strain. For this a series of experiments on a historic $19^{\text {th }}$ century tapestry inside an environmental chamber were carried out and cycles of moisture adsorption and desorption tested. Digital Image Correlation (DIC) and magnetic sensors developed by IBM were used to test successive stages of expansion and retraction of the tapestry given different levels of relative humidity (RH) in the environment.
\end{abstract}

Peer-review under the responsibility of the organizing committee of the ICMB21.

Keywords: Tapestries; Environmental testing; DIC; Strain;

\section{Introduction}

Historic tapestries are textile artworks weaved by hand with wool, silk, linen and sometimes metallic threads with the purpose of rendering an image to hang in interior spaces either for decoration and/or thermal insulation. They were extensively in use from $15^{\text {th }}$ to the $18^{\text {th }}$ century, and they adorned some of the richest buildings across Europe [1]. However, tapestries change with time because they are exposed to a range of damaging factors such as variable environmental conditions [2]. Cycles of moisture absorption and desorption make the tapestry's weight a variable over time. Furthermore, with cycles of moisture absorption and desorption the tapestry undergoes deformations which are related not only to the amount of moisture in the air but also to the type of textile fibres present in the tapestry, as they have different hygroscopic properties and different mechanical characteristics [3]. Depending on the design of the tapestries they can have areas made entirely of wool or silk, each with different rates of water absorption and mechanical response. In a tapestry structure this will create changing deformations overtime which creates differential strain in different areas of the tapestry.

\section{Methodology}

The 19th century
tapestry was initially
analyzed with an electronic
microscope where fiber
identification showed this
tapestry warp as linen and
weft a mix between wool
and silk threads. The
tapestry was hung in an
aluminum frame inside an
environmental chamber
(Figure 1 (a) and (b)) and
conditioned to a
temperature (T) of $25^{\circ} \mathrm{C}$ and
$45 \%$ of relative humidity $(\mathrm{RH})$ prior to testing.
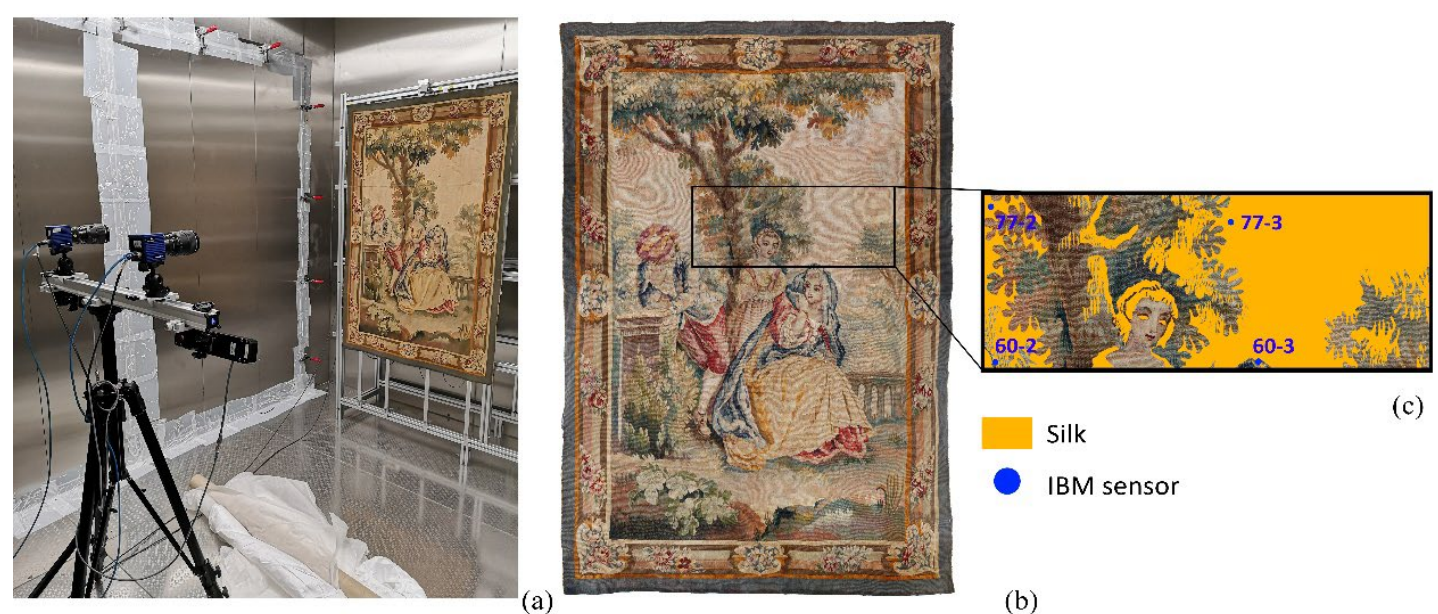

Silk

IBM sensor

(b)

Figure 1. (a) DIC camera setup inside the environmental chamber (b) Front view of the tapestry (c) Area selected for DIC analysis and distribution of IBM sensors. 
ICMB21

A two-camera stereoscopic DIC system was setup inside the chamber and calibrated to record a middle region in the tapestry that included both silk and wool as Figure 1 (c) shows. The average vertical and horizontal strains in this region were calculated on the Davis DIC software using 64 pixels as correlation subset size and a step size of 8 pixels. Smaller local displacements were measured using four IBM magnetic sensors [4] placed in specific areas of the tapestry as represented in Figure 1 (c).

The tapestry was exposed to four-hour cycles of moisture fluctuations run at a constant temperature of $25^{\circ} \mathrm{C}$ as showed in red in Figure 2. In these cycles the RH levels vary from $45 \%$ to $40 \%$, then increases to $80 \%$ and reduces to $45 \%$ again to start a new cycle, as showed in blue in Figure 2.

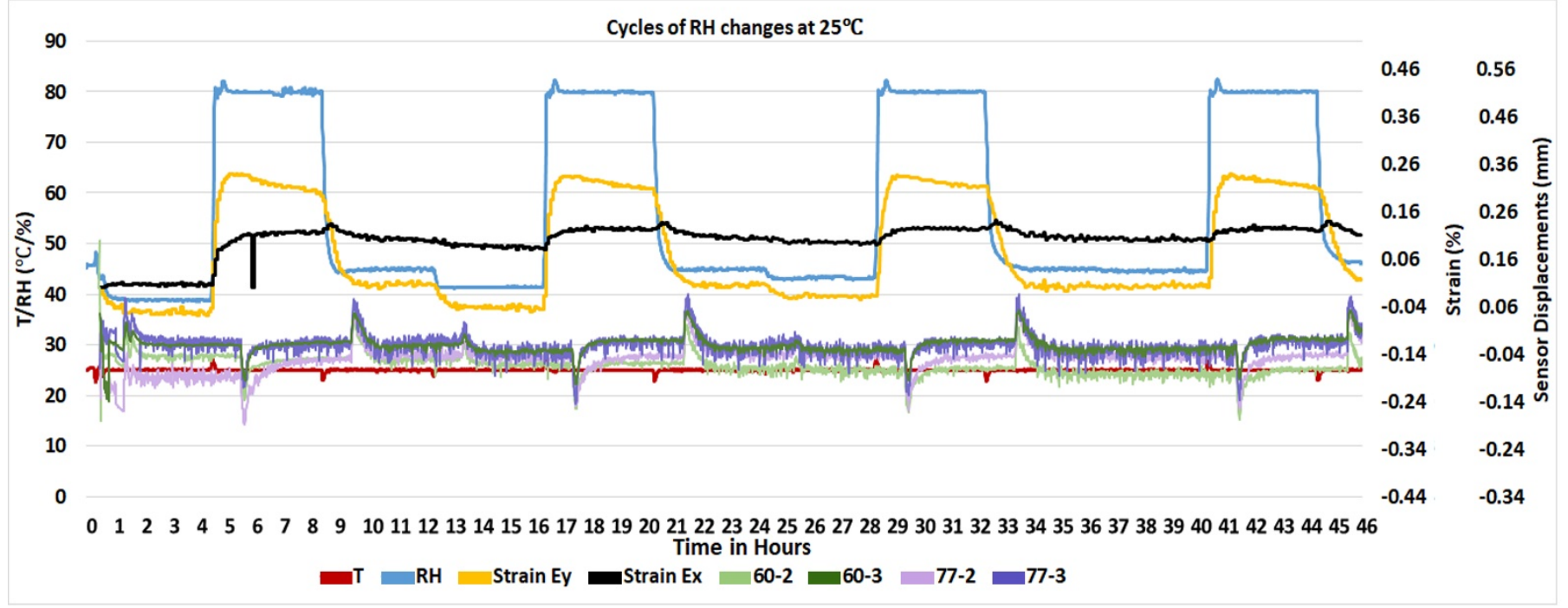

Figure 2. Cycles of change in RH, respective DIC calculated strains horizontal Ex and vertical Ey and IBM sensor displacements.

\section{Results and Discussion}

The experiment showed that the tapestry quickly adapts to the changes in moisture of the surrounding environment. As shown in Figure 2 the time for the tapestry to reach strain equilibrium is around 30 and 80 minutes when RH changes from $40 \%$ to $80 \%$ and from $80 \%$ to $45 \%$ respectively. Vertical strains undergoes higher changes than horizontal strains due to moisture cycles. Both strains follow the same behavior, although the variation in horizontal strain is very modest and delayed after the first cycle.

Although the setup for RH was the same for each cycle, it can be noted that after the first cycle the $\mathrm{RH}$ does not reach $40 \%$ (Figure 2) however it is noticeable how the vertical strain mimics the diverse amplitude of RH changes accurately. It is also noticeable that a small $5 \%$ decrease in $\mathrm{RH}$ from $45 \%$ to $40 \%$ causes a proportionally higher change in vertical strain ( $0.05 \%)$ when compared to the change of $0.2 \%$ caused by a variation in RH of $35 \%$ from 80 to $45 \%$. Overall the tapestry shows an elastic response with good repeatability of results over the cycles.

The displacements on IBM sensors (Figure 2) show an initial peak of movement of around $0.1 \mathrm{~mm}$ either negative if there is an increase in $\mathrm{RH}$ or positive if the $\mathrm{RH}$ decreases.

\section{Conclusions and Future Work}

The results suggest that strain in tapestries quickly adapts and equilibrates to the changing in the environment. This means that tapestries quickly absorb and release water changing and reaching a weight equilibrium to any fluctuation in RH. It was also shown that the tapestry takes a shorter time in adsorption than in desorption on the RH levels tested. Future experiments aim to test different levels of $\mathrm{T}$ and the effect of summer and winter day conditions found in the indoor environment of the Great Hall in Hampton Court Palace.

\section{References}

[1] Guiffrey, J. (1886). Histoire De La Tapisserie: Depuis Le Moyen Âge Jusqu'à Nos Jours. Andesite Press.

[2] Frame, K., Vlachou-Mogire, C., Hallett, K., \& Takami, M. (2018). Balancing Significance and Maintaining 'Sense of Place' in the Sustainable Display of Tudor Tapestries in the Great Hall, Hampton Court Palace. Studies in Conservation, 63(sup1), 87-93. https://doi.org/10.1080/00393630.2018.1497320

[3] Máximo-Rocha, P., D’Ayala, D., \& Vlachou-Mogire, C. (2018). Methodology for tensile testing historic tapestries. IOP Conference Series: Materials Science and Engineering, 364, 012003. https://doi.org/10.1088/1757-899X/364/1/012003

[4] Sloan, J., Klein, L., Bermudez Rodriguez, S., Hamann, H., \& Schrott, A. (2014). Concealable strain sensing method for art preservation. Applied Physics A: Materials Science and Processing, 115, 3, 829-836. https://doi.org/10.1007/s00339-013-7871-4 